\title{
Anterior hypopituitarism secondary to biopsy-proven IgG4-related hypophysitis in a young man
}

\author{
H Joshi1, M Hikmat', A P Devadass², S O Oyibo' ${ }^{1}$ and S V Sagi1 \\ 1Department of Endocrinology, Peterborough City Hospital, Peterborough, UK and 2Department of Histopathology, \\ Addenbrookes Hospital, Cambridge, UK
}

Correspondence should be addressed to S O Oyibo

Email

samoyibo@yahoo.co.uk

\section{Summary}

IgG4-related disease (IgG4-RD) is an immune-mediated fibro-inflammatory condition which can affect various organs including the pituitary gland. The true annual incidence of this condition remains widely unknown. In addition, it is unclear whether IgG4 antibodies are causative or the end result of a trigger. With no specific biomarkers available, the diagnosis of IgG4-related hypophysitis remains a challenge. Additionally, there is a wide differential diagnosis. We report a case of biopsy-proven IgG4-related hypophysitis in a young man with type 2 diabetes mellitus.

\section{Learning points:}

- IgG4-related hypophysitis is part of a spectrum of IgG4-related diseases.

- Clinical manifestations result from anterior pituitary hormone deficiencies with or without diabetes insipidus, which can be temporary or permanent.

- A combination of clinical, radiological, serological and histological evidence with careful interpretation is required to make the diagnosis.

- Tissue biopsy remains the gold standard investigation.

- Disease monitoring and long-term management of this condition is a challenge as relapses occur frequently.

\section{Background}

Immunoglobulin G4-related disease (IgG4-RD) is a relapsing and remitting fibro-inflammatory condition characterised by infiltration of tissue with plasma cells. These conglomerates of plasma cells largely secrete immunoglobulins particularly of the IgG4 subclass in significant concentrations (1). The hallmark traits of IgG4-RD are the histopathologic appearances of a dense lymphoplasmacytic infiltrate with storiform fibrosis and immunohistochemistry findings of more than ten plasma cells per high-power field with an IgG4+/IgG+ plasma cell ratio of more than $40 \%$ (2).

At present, epidemiological data are limited. To date, no biomarkers are available to fully assess the severity or monitor activity of the disease. However, the serum IgG4 level has been found to be raised in some cases and is included in the diagnostic criteria for IgG4-RD (2). Treatment is largely based on clinical experience; however, glucocorticoids have been recommended as first-line therapy with emphasis on adjunct immunosuppressive agents for relapsing cases.

Although IgG4-RD is a multi-systemic disorder, there have been cases of isolated IgG4-related hypophysitis (3). Affected patients usually present with features of anterior and/or posterior hypopituitarism. We present a patient with IgG4-related hypophysitis resulting in anterior hypopituitarism. 


\section{Case presentation}

A 39-year-old man of Asian origin, presented with a 4-week history of frontal headache and recent onset double vision. He was diagnosed with type 2 diabetes mellitus (on treatment with metformin) 1 month before and was a smoker. He had a family history of diabetes mellitus.

On clinical examination, he was overweight (weight $95 \mathrm{~kg}$, BMI 28.1). He had right-sided sixth cranial nerve palsy. No other neurological deficit was noted. Visual field assessment was normal. Because of his vascular risk factors, a brain stem ischaemic event causing internuclear ophthalmoplegia was suspected along with the possibility that this could have been a first presentation of a demyelinating disease. He was commenced on an antiplatelet agent, a lipid-lowering agent and given a 3-day course of methylprednisolone. The patient's headaches and double vision improved with treatment over the next 2 weeks. Because initial blood results revealed low testosterone levels, he was referred to the endocrine clinic for follow-up.

Three months later, he was admitted to hospital feeling generally unwell for the last few weeks with severe headaches, weight loss $(12 \mathrm{~kg})$, vomiting and double vision. He weighed $82 \mathrm{~kg}$ and visual examination did not reveal any visual impairment or ophthalmoplegia.

\section{Investigation}

The results of the laboratory investigations from his first and second presentations with normal reference levels are shown in Table 1. During his first presentation, his haematology, renal and liver function tests were normal. A pituitary profile was normal apart from mild hypogonadotrophic hypogonadism. A short Synacthen test revealed normal adrenal function (baseline cortisol: $420 \mathrm{nmol} / \mathrm{L}, 30-\mathrm{min}$ cortisol: $1130 \mathrm{nmol} / \mathrm{L})$. A computed tomography scan demonstrated a bulky pituitary gland. An MRI scan (Fig. 1A and B) reported a normal-sized pituitary gland (height: $9 \mathrm{~mm}$ ) with upward convex margin and no evidence of brain stem ischaemia. His bone density scan was normal. Cerebral spinal fluid examination showed a raised white cell count of 16 cells per microlitre predominantly comprising lymphocytes and slightly raised protein levels but no evidence of oligoclonal bands.

During his second presentation, there was clear evidence of anterior hypopituitarism with raised prolactin. There was no evidence of diabetes insipidus. Repeat MRI with contrast (Fig. 2A and B) revealed a larger and brighter pituitary mass in comparison to the first scan with suprasellar extension displacing the optic chiasm and a thickened pituitary stalk.

Further investigations revealed a normal computed tomography scan of chest, abdomen and pelvis, normal beta human chorionic gonadotrophin, serum ACE and $\alpha$-fetoprotein levels. Patient had non-reactive HIV and quantiferon gold tests, which excluded HIV and tuberculosis. Serum light-free chains and immunoglobulin levels including serum IgG4 were also normal.

A trans-sphenoidal pituitary biopsy was done a year after presentation (patient-related delay) and histological examination revealed non-adenomatous pituitary tissue with a sclerosing chronic inflammatory infiltrate of plasma cells. The immunohistochemistry examination demonstrated 18 IgG4+ plasma cells per high-power field with an IgG4+/IgG+ plasma cell ratio of $45 \%$, suggestive of IgG4-related hypophysitis (Fig. 3A, B and Table 2).

\section{Treatment}

The patient was treated with physiological-dose hydrocortisone for the hypocortisolaemia followed by levothyroxine and testosterone replacement. He was also

Table 1 Laboratory results with normal values at presentation and 3 years later.

\begin{tabular}{l}
\hline Parameter \\
\hline Luteinizing hormone \\
Follicle-stimulating hormone \\
Free thyroxine \\
Free triiodothyronine \\
Thyroid-stimulating hormone \\
Insulin growth factor 1 \\
Early morning cortisol \\
Testosterone \\
Prolactin \\
Immunoglobulin subclass 4 (IgG4)
\end{tabular}

https://edm.bioscientifica.com/

\begin{tabular}{ccc}
\hline Normal serum values & & 1st presentation \\
\cline { 1 - 1 } $2-9 \mathrm{U} / \mathrm{L}$ & & 2 \\
$2-13 \mathrm{U} / \mathrm{L}$ & & 2 \\
$12.0-22.0 \mathrm{pmol} / \mathrm{L}$ & & 20.4 \\
$3.1-6.8 \mathrm{pmol} / \mathrm{L}$ & & - \\
$0.30-0.52 \mathrm{mU} / \mathrm{L}$ & & 1.26 \\
$16.3-39.3 \mathrm{nmol} / \mathrm{L}$ & & 33.0 \\
$\mathrm{nmol} / \mathrm{L}$ & & 424 \\
$10.0-38.0 \mathrm{nmol} / \mathrm{L}$ & & 7.4 \\
$<330 \mathrm{mU} / \mathrm{L}$ & 260 \\
$0.0-1.3 \mathrm{~g} / \mathrm{L}$ & \\
\hline
\end{tabular}

\begin{tabular}{c}
\hline 2nd presentation \\
\hline$<1.0$ \\
$<1.0$ \\
7.2 \\
- \\
0.03 \\
9.8 \\
47 \\
1.3 \\
790 \\
0.33 \\
\hline
\end{tabular}

\begin{tabular}{c}
\hline 3 years after presentation \\
\hline$<1.0$ \\
$<1.0$ \\
15.9 \\
4.7 \\
0.63 \\
22.6 \\
- \\
15.3 \\
169 \\
0.24
\end{tabular}



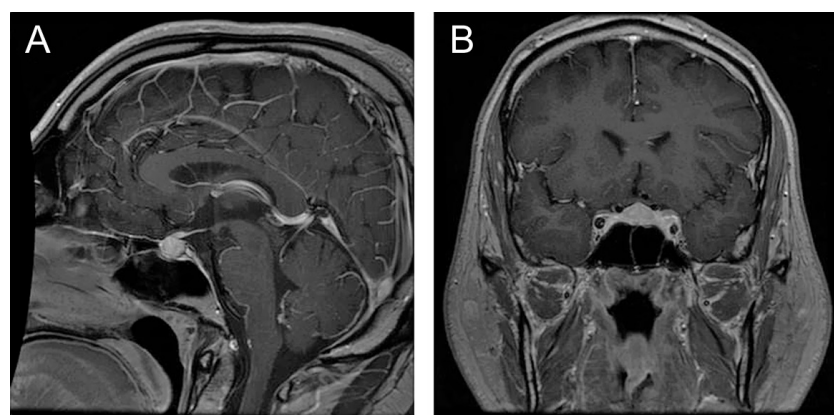

Figure 1

(A and B) Images demonstrate a pituitary gland (height $9 \mathrm{~mm}$ ) with convex upward margin and enhancement of the pituitary stalk. (A) Sagittal MRI view at first presentation. (B) Coronal MRI view at first presentation.

found to be deficient in vitamin $\mathrm{D}$ and was initiated on an appropriate dose.

\section{Outcome and follow-up}

The results were discussed with the patient. The use of high-dose glucocorticoid therapy was discussed with the Pituitary Multidisciplinary Team. As it was a year after presentation and fibrosis had already set in, it was decided we continue observation. The patient's headaches and visual symptoms resolved once he was commenced on physiological-dose glucocorticoid replacement at second presentation, but he still had intermittent headaches. His general health improved over 6 months, as indicated by a return to his usual (pre-illness) weight. His repeat MRI scans demonstrated gradual improvement over the next 3 years (Fig. 4A and B). Serial pituitary function tests after diagnosis showed no resolution of the pituitary dysfunction. To date, the patient remains clinically well on anterior pituitary hormone replacement therapy.
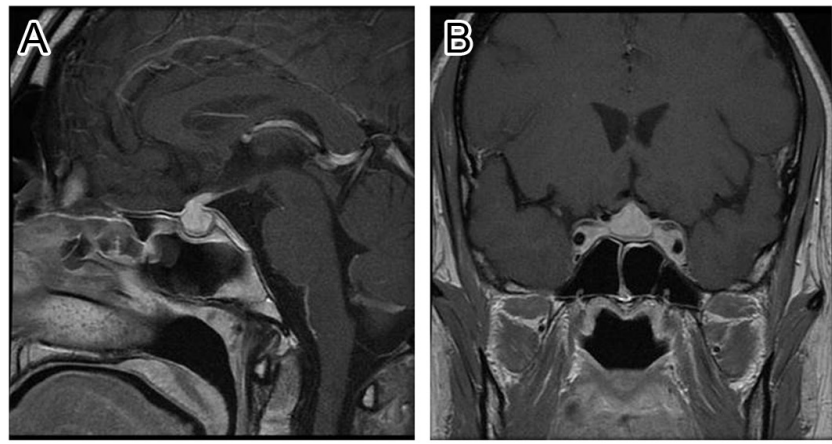

Figure 2

(A and B) Images demonstrate superior convexity of the pituitary gland very close to the optic chiasm and a thickened pituitary stalk. (A) Sagittal MRI view at second presentation. (B) Coronal MRI view at second presentation.
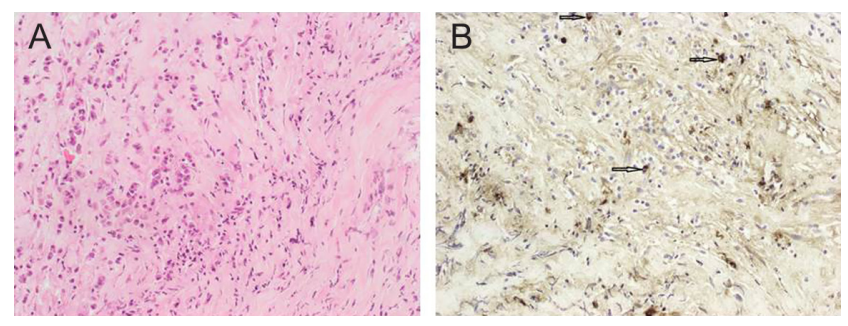

\section{Figure 3}

(A) Image demonstrates dense fibrous tissue sparsely infiltrated by cytologically bland plasma cells at overall low packing-density, but no obvious granulomata, eosinophils, polymorphs or lymphocytes. (B) Image demonstrates a significant fraction of the plasma cells that are immunoreactive for IgG4 (arrows). (A) Biopsy specimen cytology (×200). (B) Biopsy specimen immunohistochemistry $(\times 200)$.

\section{Discussion}

IgG4-RD can involve single or multiple organs leading to a wide spectrum of clinical manifestations. Isolated involvement of the pituitary gland is termed 'hypophysitis'. This can be classified based on aetiology (primary or secondary), morphology (adenohypophysitis, infundibulo-neurohypophysitis or both) and/or histopathology (autoimmune, granulomatous, xanthomatous, necrotising or plasmacyctic/IgG4-related). IgG4-related hypophysitis is rare and constitutes $1.3 \%$ of all primary hypophysitis cases (4).

Epidemiological data for Ig4-related disease is limited. A recent review of 84 patients with IgG4-related hypophysitis showed a male predominance of 2:1 with a mean age of onset at 64 years. Elevated serum IgG4 levels ( $>140 \mathrm{mg} / \mathrm{dL}$ ) were found in $80 \%$ of the patients. Fortyone patients had anterior pituitary dysfunction and 39 patients had diabetes insipidus (4). The pathogenic role of IgG4 remains intriguing to date. IgG4 is in fact viewed as an anti-inflammatory molecule and is the least common subtype with concentrations less than 5\% of total IgG. The IgG4 subclass has a comparatively lower affinity

Table 2 Histology report.

\begin{tabular}{|c|c|}
\hline Parameter & Results \\
\hline Tissue & $\begin{array}{l}\text { Non-adenomatous pituitary } \\
\text { sample }\end{array}$ \\
\hline P53 & Negative \\
\hline CD79a (B cell marker) & Scanty B cells \\
\hline CD3 ( $\mathrm{T}$ cell marker) & Scanty T cells \\
\hline CD68P (Macrophage marker) & Scanty macrophages \\
\hline IgG (Pan Immunoglobulin G) & Several reactive plasma cells \\
\hline IgG4+ plasma cells & 18 positive plasma cells per HPF \\
\hline IgG4+/lgG plasma cell ratio & $0.45(45 \%)$ \\
\hline Analysis & $\begin{array}{l}\text { Sclerosing chronic inflammation } \\
\text { compatible with IgG4 RD }\end{array}$ \\
\hline
\end{tabular}



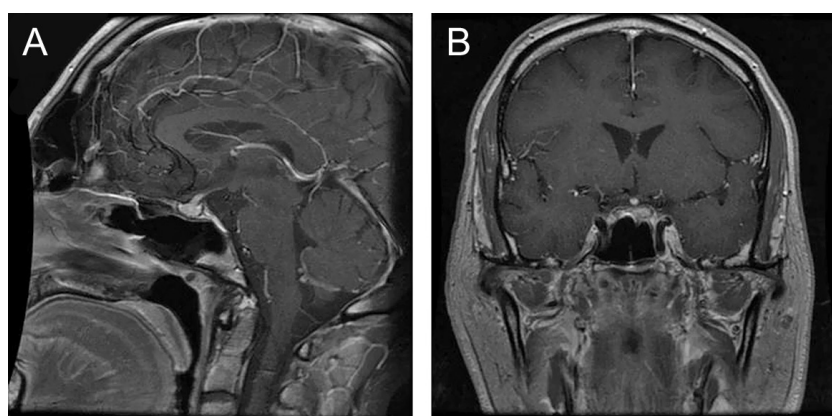

Figure 4

(A and B) Images demonstrate enhancement of the pituitary stalk and peripheral enhancement of the gland, but if anything this is a fraction smaller in height, suggesting slight regression. (A) Sagittal MRI view 3 years after initial presentation. (B) Coronal MRI view 3 years after initial presentation.

and therefore is not seen to be effective in initiating complement reactions (5). Evidence concerning the true driver of inflammation is still lacking.

In hypophysitis, patients usually present with symptoms from mass effect and/or hormonal symptoms depending on the anatomical region and extent of involvement. Several diagnostic criteria for IgG4-related hypophysitis have been put forward. One example involves the use of five clinical features in different combinations namely the presence of mononuclear cell infiltrates rich in plasma cells and lymphocytes with more than ten IgG4-positive cells per high-power field; a sellar mass and or thickened stalk; biopsy-proven involvement with IgG4 staining; raised serum IgG4 levels $>140 \mathrm{mg} /$ $\mathrm{dL}$ and reduction in sellar mass size with symptom improvement after treatment with glucocorticoids (3). Another diagnostic criterion has a lower cut-off level for serum IgG4 $(135 \mathrm{mg} / \mathrm{dL})$ and also included immunohistochemistry (IgG4+/IgG+ plasma cell ratio of more than 40\%) (6). Both diagnostic criteria highlight the importance of the diagnostic limitations of serum IgG4 levels, which can vary between systemic and organspecific IgG4-RD.

Imaging methods such as computed tomography and magnetic resonance imaging are useful for outlining the extent of pathological involvement and to assess if surgical intervention is needed. F-fluorodeoxyglucose positron emission tomography or computed tomography (FDG-PET-CT) is now being considered as an effective modality for the diagnosis of IgG4-related disorders (7).

Steroids are the mainstay of treatment, and a rapid response is usually evident. However, there is a risk of steroid dependency and possible relapse on a tapering steroid dose. High-dose methylprednisolone therapy as treatment for lymphocytic hypophysitis has been advocated as a high-dose, short-term way to administer methylprednisolone while minimising unwanted side effects of chronic corticosteroid therapy (8). Methotrexate, mycophenolate mofetil and rituximab are a few of the steroid-sparing agents which are being used with variable efficacy $(9,10)$.

Our patient first presented with headache and double vision. The fact that he had diabetes mellitus and was a smoker widened the differential diagnosis. It is likely that the superior convex margin of the pituitary gland demonstrated on MRI scan at first presentation represented hypophysitis at that time. On hindsight, the pituitary height was slightly abnormal. It is important to note that changes in pituitary height vary in different age groups of both genders. In a study involving 500 patients of Asian background, a pituitary height of more than $8 \mathrm{~mm}$ in the 31-40 years age group of male gender was considered abnormal (11). Pituitary hormone profile was normal apart from his testosterone level, which was suppressed. His second presentation with pituitary failure prompted further investigations which yielded the characteristic pituitary image and biopsy-proven result. There has been demonstrable and continued resolution of the IgG4-related pituitary inflammation without the need for prolonged use of supraphysiological-dose glucocorticoid treatment. We suspect that the temporary sixth nerve palsy was due to IgG4-related infiltration rather than due to diabetes-related mononeuropathy, as his glycated haemoglobin value was $68 \mathrm{mmol} / \mathrm{mol}$ and he had no evidence of long-term microvascular complications. Additionally, he had complete resolution of the sixth cranial nerve palsy after methylprednisolone at initial presentation. Another important fact is that this patient was initially reluctant to have a biopsy. After several months of informed discussion, he requested a referral to another tertiary centre for a second opinion. Therefore, he had the biopsy nearly a year after initial presentation. Had the biopsy been performed earlier to confirm the diagnosis, the patient would have started supraphysiological-dose glucocorticoids. This may have led to an earlier remission of the disease. Furthermore, the multidisciplinary team's rationale for doing the biopsy was to assess whether this was an inflammatory disorder or an infiltrative disorder.

This case is unique for several reasons. Firstly, our patient is much younger than those reported in several case reports where the mean \pm SD age of onset is $64.2 \pm 13.9$ years (4). Secondly, most cases had raised serum IgG4 levels but in our patient's case the serum IgG4 
level was normal, highlighting the fact that serum IgG4 levels is not required for diagnosis. Thirdly, up to $70 \%$ of cases have diabetes insipidus associated with them, but our patient did not have any evidence of diabetes insipidus. Fourthly, not many cases have histopathological confirmation of diagnosis.

In conclusion, we report a case of IgG4-related hypophysitis in a young man in whom the diagnosis was confirmed histologically. Notably, the disease was specific to the pituitary gland resulting in anterior hypopituitarism and there was gradual resolution of the pituitary lesion without the prolonged use of supraphysiological glucocorticoids. We hope that this case will not only add to the existing literature but also contribute to the ongoing characterisation and management of this complex disease.

\section{Declaration of interest}

The authors declare that there is no conflict of interest that could be perceived as prejudicing the impartiality of the research reported.

\section{Funding}

This research did not receive any specific grant from any funding agency in the public, commercial or not-for-profit sector.

\section{Patient consent}

Written informed consent has been obtained from the patient for publication of the submitted article and any accompanying images.

\section{Author contribution statement}

$\mathrm{H}$ Joshi, M Hikmat, A P Devadass, S O Oyibo and S V Sagi all wrote the case report and critically revised the paper and approved the final manuscript for submission. A P Devadass also provided assistance with the histology and immunohistochemistry presentation. S V Sagi identified the case and is also the named physician of the patient.

\section{References}

1 Stone JH, Zen Y \& Deshpande V. VIgG4-Related disease. New England Journal of Medicine 2012366 539-551. (https://doi.org/10.1056/ NEJMra1104650)

2 Lang D, Zwerina J \& Pieringer H. IgG4-related disease: current challenges and future prospects. Therapeutics and Clinical Risk Management 201612 189-199. (https://doi.org/10.2147/TCRM. S99985)

3 Leporati P, Landek-Salagado MA, Lupi I, Chiovato L \& Caturegli P. IgG4-related hypophysitis: a new addition to the hypophysitis spectrum. Journal of Clinical Endocrinology and Metabolism 201196 1971-1980. (https://doi.org/10.1210/jc.2010-2970)

4 Shikuma J, Kan K, Ito R, Hara K, Sakai H, Miwa T, Kanazawa A \& Odawara M. Critical review of IgG4-related hypophysitis. Pituitary 201720 282-291. (https://doi.org/10.1007/s11102-016-0773-7)

5 Weindorf SC \& Frederiksen JK. IgG4-related disease: a reminder for practicing pathologists. Archives of Pathology and Laboratory Medicine 2017141 1476-1483. (https://doi.org/10.5858/arpa.20170257-RA)

6 Umehara H, Okazaki K, Masaki Y, Kawano M, Yamamoto M, Saeki T, Matsui S, Sumida T, Mimori T, Tanaka Y, et al. A novel clinical entity, IgG4-related disease (IgG4RD): general concept and details. Modern Rheumatology 201222 1-14. (https://doi.org/10.1007/s10165-0110508-6)

7 Zhang J, Chen H, Ma Y, Xiao Y, Niu N, Lin W, Wang X, Liang Z, Zhang F, Li F, et al. Characterizing IgG4-related disease with 18FFDG PET/CT: a prospective cohort study. European Journal of Nuclear Medicine and Molecular Imaging 201441 1624-1634. (https://doi. org/10.1007/s00259-014-2729-3)

8 Kristof RA, Van Roost D, Klingmuller D, Springer W \& Schramm J. Lymphocytic hypophysitis: non-invasive diagnosis and treatment by high dose methylprednisolone pulse therapy? Journal of Neurology, Neurosurgery and Psychiatry 199967 398-402. (https://doi. org/10.1136/jnnp.67.3.398)

9 Khosroshahi A, Wallace ZS, Crowe JL, Akamizu T, Azumi A, Carruthers MN, Chari ST, Della-Torre E, Frulloni L, Goto H, et al. International consensus guidance statement on the management and treatment of IgG4-related disease. Arthritis and Rheumatology 201567 1688-1699. (https://doi.org/10.1002/art.39132)

10 Karim AF, Bansie RD, Rombach SM, Paridaens D, Verdijk RM, van Hagen PM \& van Laar JAM. The treatment outcomes in IgG4-related disease. Netherlands Journal of Medicine 201876 275-285.

11 Yadav P, Singhal S, Chauhan S \& Harit S. MRI evaluation of size and shape of normal pituitary gland: age and sex related changes. Journal of Clinical and Diagnostic Research 201712 1-4. (https://doi. org/10.7860/JCDR/2017/31034.10933)

Received in final form 13 February 2019

Accepted 18 March 2019 